\title{
A CLASS OF UNIVALENT FUNCTIONS
}

\author{
T. R. CAPLINGER AND W. M. CAUSEY
}

Abstract. A sharp coefficient estimate is obtained for a class $D(\alpha)$ of functions univalent in the open unit disc. The radius of convexity and an arclength result are also determined for the class.

Let $D(\alpha)$ denote the class of functions $f(z)=z+a_{2} z^{2}+\cdots$ analytic in the open unit disc $E$ and satisfying

$$
\left|\left(f^{\prime}(z)-1\right) /\left(f^{\prime}(z)+1\right)\right|<\alpha, \quad z \in E,
$$

for some $\alpha, 0<\alpha \leqq 1$. The values $f^{\prime}(z)$ lie inside the circle in the right half plane with center $\left(1+\alpha^{2}\right) /\left(1-\alpha^{2}\right)$ and radius $2 \alpha /\left(1-\alpha^{2}\right)$. The class $D(\alpha)$ is a subclass of the class of functions whose derivative has positive real part and hence a function in $D(\alpha)$ is univalent in $E$. If $f \in D(\alpha)$ it follows from Schwarz lemma that $f^{\prime}(z)=(1-\alpha z \theta(z)) /(1+\alpha z \theta(z))$, where $\theta(z)$ is analytic and $|\theta(z)| \leqq 1$ in $E$.

A class of starlike functions has been studied by Padmanabhan [5] in which $f^{\prime}(z)$ is replaced by $z f^{\prime}(z) / f(z)$ in inequality (1).

A sharp coefficient estimate for the class $D(\alpha)$ is proved in Theorem 1 using a technique of Clunie and Keogh [2]. In Theorem 2 the radius of convexity of the class is obtained and in Theorem 3 an arclength result is given.

THEOREM 1. If $f(z)=z+\sum_{n=2}^{\infty} a_{n} z^{n}$ is in $D(\alpha)$ for some $\alpha, 0<\alpha \leqq 1$, then $\left|a_{n}\right| \leqq 2 \alpha / n, n=2,3, \cdots$. The inequality is sharp.

Proof. Since $f(z)$ is in $D(\alpha)$, then $f^{\prime}(z)=(1+\alpha z \theta(z)) /(1-\alpha z \theta(z))$, where $\theta(z)=\sum_{n=1}^{\infty} t_{n} z^{n}$ is analytic and $|\theta(z)| \leqq 1$ for $z \in E$. Then

or

$$
f^{\prime}(z)-1=\alpha z \theta(z)\left\{f^{\prime}(z)+1\right\},
$$

$$
\sum_{n=2}^{\infty} n a_{n} z^{n-1}=\alpha\left(\sum_{n=0}^{\infty} t_{n} z^{n}\right)\left(2 z+\sum_{n=2}^{\infty} n a_{n} z^{n}\right) .
$$

Equating corresponding coefficients in (2) gives

$$
n a_{n}=\alpha\left\{(n-1) t_{0} a_{n-1}+(n-2) t_{1} a_{n-2}+\cdots+2 t_{n-3} a_{2}+2 t_{n-2}\right\} .
$$

Presented to the Society, January 26, 1973; received by the editors September 5, 1972 and, in revised form, September 25, 1972.

AMS (MOS) subject classifications (1970). Primary 30A32, 30A34.

Key words and phrases. Univalent, convex.

(c) American Mathematical Society 1973 
Thus $a_{n}$ depends only on $a_{2}, a_{3}, \cdots, a_{n-1}$ and $\theta$ for $n \geqq 2$. Hence, for $n \geqq 2$, it follows from (2) that

$$
\sum_{k=2}^{n} k a_{k} z^{k-1}+\sum_{k=n+1}^{\infty} b_{k} z^{k-1}=\alpha \theta(z)\left\{2 z+\sum_{k=2}^{n-1} k a_{k} z^{k}\right\},
$$

which yields

$$
\begin{aligned}
\left|\sum_{k=2}^{n} k a_{k} z^{k-1}+\sum_{k=n+1}^{\infty} b_{k} z^{k-1}\right|^{2} & =\alpha^{2}|\theta(z)|^{2}\left|2 z+\sum_{k=2}^{n-1} k a_{k} z^{k}\right|^{2} \\
& \leqq \alpha^{2}\left|2 z+\sum_{k=2}^{n-1} k a_{k} z^{k}\right|^{2} .
\end{aligned}
$$

Integrating about $|z|=r, 0<r<1$, gives

$$
\sum_{k=2}^{n} k^{2}\left|a_{k}\right|^{2} r^{2 k-2}+\sum_{k=n+1}^{\infty}\left|b_{k}\right|^{2} r^{2 k-2} \leqq \alpha^{2}\left\{4 r^{2}+\sum_{k=2}^{n-1} k^{2}\left|a_{k}\right|^{2} r^{2 k}\right\} .
$$

If we take the limit as $r$ approaches 1, then

or

$$
\sum_{k=2}^{n} k^{2}\left|a_{k}\right|^{2} \leqq \alpha^{2}\left\{4+\sum_{k=2}^{n-1} k^{2}\left|a_{k}\right|^{2}\right\},
$$

$$
\begin{aligned}
n^{2}\left|a_{n}\right|^{2} & \leqq 4 \alpha^{2}+\alpha^{2} \sum_{k=2}^{n-1} k^{2}\left|a_{k}\right|^{2}-\sum_{k=2}^{n-1} k^{2}\left|a_{k}\right|^{2} \\
& =4 \alpha^{2}+\left(\alpha^{2}-1\right) \sum_{k=2}^{n-1} k^{2}\left|a_{k}\right|^{2} \leqq 4 \alpha^{2},
\end{aligned}
$$

since $\alpha \leqq 1$. Thus $\left|a_{n}\right| \leqq 2 \alpha / n$ for $n \geqq 2$.

Sharpness of the inequality is shown by

$$
f(z)=\int_{0}^{z} \frac{1+\alpha t^{n-1}}{1-\alpha t^{n-1}} \alpha t=z+\frac{2 \alpha z^{n}}{n}+\frac{2 \alpha^{2}}{2 n-1} z^{2 n-1}+\cdots .
$$

THEOREM 2. If $f(z)$ is in $D(\alpha), 0<\alpha \leqq 1$, then

(i) $f(z)$ maps $|z|<(\sqrt{ } 2-1) / \alpha$ onto a convex domain if

(ii) $f(z)$ maps

$$
\left(\frac{(\sqrt{ } 2-1)(\sqrt{ } 3+1)}{\sqrt{ } 2}\right) \leqq \alpha \leqq 1,
$$

$$
|z|<\left[\left\{\alpha^{2}-1+\left(\left(1-\alpha^{2}\right)\left(1+4 \alpha-\alpha^{2}\right)\right)^{1 / 2}\right\} / 2 \alpha(1+\alpha)\right]^{1 / 2}
$$

onto a convex domain if

$$
0<\alpha \leqq(\sqrt{ } 2-1)(\sqrt{ } 3+1) / \sqrt{ } 2 .
$$

The bounds in (i) and (ii) are both sharp. 
Proof. Since $f(z)$ is in $D(\alpha)$, we have

$$
f^{\prime}(z)=(1+\alpha z \theta(z)) /(1-\alpha z \theta(z)),
$$

where $\theta(z)$ is analytic and $|\theta(z)| \leqq 1$ for $z \in E$. Then

$$
\frac{f^{\prime \prime}(z)}{f^{\prime}(z)}=\frac{2 \alpha\left\{z \theta^{\prime}(z)+\theta(z)\right\}}{1-\alpha^{2} z^{2}[\theta(z)]^{2}} .
$$

But for functions $\theta(z)[4$, p. 168] we have

$$
\left|\theta^{\prime}(z)\right| \leqq\left(1-|\theta(z)|^{2}\right) /\left(1-|z|^{2}\right) .
$$

Using this estimate we obtain

$$
\left|\frac{z f^{\prime \prime}(z)}{f^{\prime}(z)}\right| \leqq \frac{2 \alpha|z|(|z|+|\theta(z)|)(1-|z \theta(z)|)}{\left(1-|z|^{2}\right)\left(1-\alpha^{2}|z|^{2}|\theta(z)|^{2}\right)} .
$$

Therefore, $\left|z f^{\prime \prime}(z)\right| f^{\prime}(z) \mid \leqq 1$ provided

(3) $2 \alpha|z|(|z|+|\theta(z)|)(1-|z \theta(z)|) \leqq\left(1-|z|^{2}\right)\left(1-\alpha^{2}|z|^{2}|\theta(z)|^{2}\right)$.

Letting $|z|=r,|\theta(z)|=x$ and $t=r x$, relation (3) becomes

$$
2 \alpha r\left(r+t r^{-1}\right)(1-t) \leqq\left(1-r^{2}\right)\left(1-\alpha^{2} t^{2}\right) .
$$

We want to find the largest value of $\rho$ such that (3) holds for all $z$ such that $|z|<\rho$ and for all $\theta(z),|\theta(z)| \leqq 1$. This corresponds to finding the largest value of $r$ for which (4) holds for all $t, 0 \leqq t \leqq r$. Relation (4) becomes

$$
H(t) \equiv\left(\alpha^{2} r^{2}-\alpha^{2}+2 \alpha\right) t^{2}+2 \alpha\left(r^{2}-1\right) t+\left(1-2 \alpha r^{2}-r^{2}\right) \geqq 0 .
$$

We want to determine the largest value of $r$ for which $H(t) \geqq 0,0 \leqq t \leqq r$. Then $f(z)$ will map $|z|<r$ onto a convex domain. Since $H^{\prime}\left(t^{*}\right)=0$ for $t^{*}=\left(1-r^{2}\right) /\left[2-\alpha\left(1-r^{2}\right)\right]$ and $H^{\prime \prime}(t)>0, H(t)$ assumes its minimum value at $t^{*}$. We separate the proof into two cases:

Case A. $r<t^{*}$. Now $H(t)$ is nonincreasing on $[0, r]$, so $H(t)>H(r)$ for $0 \leqq t \leqq r$. Since $H(r)=\left(1-r^{2}\right)\left(-\alpha^{2} r^{2}-2 \alpha r+1\right), H(r) \geqq 0$, provided $\alpha^{2} r^{2}+2 \alpha r-1 \leqq 0$ or $r \leqq(\sqrt{ } 2-1) / \alpha$. Thus, $f(z)$ maps $|z|<(\sqrt{ } 2-1) / \alpha$ onto a convex domain if $r=(\sqrt{ } 2-1) / \alpha \leqq t^{*}$. This restraint implies that $\alpha$ must lie in the interval $\left[\alpha_{0}, 1\right]$, where $\alpha_{0} \equiv(\sqrt{ } 2-1)(\sqrt{ } 3+1) / \sqrt{ } 2$.

The function $f(z)=-z-2 / \alpha \log (1-\alpha z)$ shows this bound to be the best possible since

for $z=(1-\sqrt{ } 2) / \alpha$.

$$
1+\frac{z f^{\prime \prime}(z)}{f^{\prime}(z)}=\frac{1+2 \alpha z-\alpha^{2} z^{2}}{1-\alpha^{2} z^{2}}=0
$$


Case B. $t^{*} \leqq r$. The minimum value of $H(t)$ on $[0, r]$ occurs at $t^{*}$, so $H(t) \geqq H\left(t^{*}\right)$. Therefore (5) will be satisfied if $H\left(t^{*}\right) \geqq 0$. This inequality reduces to

$$
Q(r) \equiv \alpha(1+\alpha) r^{4}+\left(1-\alpha^{2}\right) r^{2}-(1-\alpha) \leqq 0 .
$$

But (6) is satisfied for $r<r_{1}$, where

$$
r_{1} \equiv\left[\left\{\alpha^{2}-1+\left(\left(1-\alpha^{2}\right)\left(1+4 \alpha-\alpha^{2}\right)\right)^{1 / 2}\right\} / 2 \alpha(1+\alpha)\right]^{1 / 2} .
$$

We shall show that if $0<\alpha<\alpha_{0}$, then $t^{*} \leqq r_{1}$. But $t^{*} \leqq r$, if and only if $P(r) \equiv \alpha r^{3}+r^{2}+(2-\alpha) r-1 \geqq 0$. Denote the zero of $P(r)$ in $(0,1)$ by $r_{0}$. We shall show that $r_{1}>r_{0}$ if $0<\alpha<\alpha_{0}$. A tedious calculation shows that if $\alpha=\alpha_{0}$,

$$
r_{1}=\sqrt{ } 2 /(1+\sqrt{ } 3)=r_{0}=(\sqrt{ } 2-1) / \alpha_{0} .
$$

Also for a fixed $r$ and $\alpha<\alpha_{0}$, the expression $Q(r)$ increases with $\alpha$. Thus, if (6) holds for a certain interval of values of $r$ with $\alpha=\alpha_{1}$, then the condition holds for all $\alpha<\alpha_{1}$. Hence $r_{1}$ increases with decreasing $\alpha$. But $\alpha=\alpha_{0}$ corresponds to $r_{1}=r_{0}$. Thus for $\alpha<\alpha_{0}, r_{1}>r_{0}$.

To show the estimate is sharp, we construct a function as follows. Let $\beta$ be defined by

$$
r_{1}\left(r_{1}-\beta\right) /\left(1-\beta r_{1}\right)=\left(1-r_{1}^{2}\right) /\left[2-\alpha\left(1-r_{1}^{2}\right)\right] .
$$

Since $r_{1}>r_{0}$ for $\alpha<\alpha_{0}$, it follows that $\left(1-r_{1}^{2}\right) /\left[2-\alpha\left(1-r_{1}^{2}\right)\right]<r_{1}$. Also, $r_{1}<1$. From (7) we have

or

$$
0<r_{1}\left(r_{1}-\beta\right) /\left(1-\beta r_{1}\right)<r_{1},
$$

$$
\left(r_{1}-\beta\right) /\left(1-\beta r_{1}\right)<1 \text {. }
$$

This implies that $\left(r_{1}^{2}-1\right)\left(1-\beta^{2}\right)<0$. But $r_{1}<1$, so $|\beta|<1$. Define $\theta(z)$ by

$$
\theta(z)=(z-\beta) /(1-\beta z) \text {. }
$$

Since $|\beta|<1,|\theta(z)| \leqq 1$ for $z \in E$. Define $f(z)$ by

Then

$$
f(z)=[1-\alpha z \theta(z)] /[1+\alpha z \theta(z)] .
$$

where

$$
1+\frac{z f^{\prime \prime}(z)}{f^{\prime}(z)}=\frac{1-\alpha^{2} z^{2}[\theta(z)]^{2}-2 \alpha z \theta(z)-2 \alpha z^{2} \theta^{\prime}(z)}{1-\alpha^{2} z^{2}[\theta(z)]^{2}},
$$

$$
\theta^{\prime}(z)=\left\{1-[\theta(z)]^{2}\right\} /\left\{1-z^{2}\right\} .
$$

Using (7) and (8) and remembering that $Q\left(r_{1}\right)=0$, we have that $1+$ $r_{1} f^{\prime \prime}\left(r_{1}\right) \mid f^{\prime}\left(r_{1}\right)=0$. Therefore, $f(z)$ is not convex in $|z|<r$ if $r>r_{1}$. 
THEOREM 3. If $f(z)$ is in $D(\alpha)$ and if $L_{r}(f)$ denotes the length of the image of $|z|=r$ under $f(z), 0<r<1$, then $L_{r}(f)=O\{\log (1 /(1-\alpha r))\}$, as $r \rightarrow 1$.

ProOF.

$$
\begin{aligned}
L_{r}(f) & =\int_{|z|=r}\left|f^{\prime}(z)\right||d z|=\int_{0}^{2 \pi}\left|f^{\prime}\left(r e^{i \theta}\right)\right| r d \theta \\
& \leqq \int_{0}^{2 \pi}\left|\frac{1+\alpha r e^{i \theta} \mid}{1-\alpha r^{i \theta}}\right| r d \theta \\
& =\int_{0}^{2 \pi}\left|\frac{1-\alpha^{2} r^{2}+2 \alpha r i \sin \theta}{1-2 \alpha r \cos \theta+\alpha^{2} r^{2}}\right| r d \theta \\
& \leqq r \int_{0}^{2 \pi} \frac{1-(\alpha r)^{2}}{1-2 \alpha r \cos \theta+(\alpha r)^{2}} d \theta+\int_{0}^{2 \pi} \frac{2 \alpha r^{2}|\sin \theta|}{1-2 \alpha r \cos \theta+\alpha^{2} r^{2}} d \theta \\
& =2 \pi r+2 r \int_{0}^{\pi} \frac{2 \alpha r \sin \theta}{1-2 \alpha r \cos \theta+\alpha^{2} r^{2}} d \theta \\
& =2 \pi r+4 r \log \frac{1+\alpha r}{1-\alpha r}=O\{\log 1 /(1-\alpha r)\} .
\end{aligned}
$$

The first integral in (9) is a Poisson integral and the second can be evaluated directly.

Remark. We state without proof that if $f(z) \in D(\alpha)$ and if $A_{r}(f)$ denotes the area of the image of $|z|<r$ under $f(z), 0<r<1$, then

$$
A_{r}(f) \leqq \pi\left\{-3-\left(4 / \alpha^{2} r^{2}\right) \log \left(1-\alpha^{2} r^{2}\right)\right\} .
$$

The inequality is sharp.

\section{REFERENCES}

1. C. Carathéodory, Funktionentheorie. Vol. II, Verlag Birkhäuser, Basel, Switzerland, 1950. MR 12, 248.

2. J. Clunie and F. R. Keogh, On starlike and convex schlicht functions, J. London Math. Soc. 35 (1960), 229-233. MR 22 \#1682.

3. T. H. MacGregor, Functions whose derivative has a positive real part, Trans. Amer. Math. Soc. 104 (1962), 532-537. MR 25 \#4090.

4. Z. Nehari, Conformal mapping, McGraw-Hill, New York, 1952. MR 13, 640.

5. K. S. Padmanabhan, On certain classes of starlike functions in the unit disk, Indian Math. Soc. J. 32 (1968), 89-103. MR 39 \#2965.

Department of Mathematics, Memphis State University, Memphis, Tennessee 38152

Department of Mathematics, University of MississipPi, University, MississipPi 38677 